\title{
Digital Analysis of BCL2 Expression in Laryngeal Squamous Cell Carcinoma
}

\author{
ARISTEIDIS CHRYSOVERGIS ${ }^{1}$, VASILEIOS S. PAPANIKOLAOU ${ }^{1}$, EVANGELOS TSIAMBAS ${ }^{2}$, \\ VASILEIOS RAGOS ${ }^{3}$, DIMITRIOS PESCHOS ${ }^{4}$ and EFTHYMIOS KYRODIMOS ${ }^{1}$ \\ ${ }^{1}$ First ENT Department, Hippocration Hospital, University of Athens, Athens, Greece; \\ ${ }^{2}$ Department of Immunohistochemistry and Molecular Biology, 401 General Army Hospital, Athens, Greece; \\ ${ }^{3}$ Department of Maxillofacial Surgery, Medical School, University of Ioannina, Ioannina, Greece; \\ ${ }^{4}$ Department of Physiology, Medical School, University of Ioannina, Ioannina, Greece
}

\begin{abstract}
Background: Deregulation of apoptosis is critical regarding the development and progression of malignancies, including laryngeal squamous cell carcinoma (LSCC). B-Cell lymphoma 2 (BCL2) (gene locus:18q21.33), located on the outer mitochondrial membrane, acts mainly as an antiapoptotic factor suppressing and blocking apoptotic signal transduction. Materials and Methods: Fifty $(n=50)$ primary LSCC tissue sections were used. Immunohistochemistry and digital image analysis were implemented for evaluating BCL2 protein expression levels. Results: High BCL2 protein expression levels were observed in 21/50 (42\%) LSCC tissue sections, whereas the remaining cases $(n=29)$ demonstrated a low expression. Overall, BCL2 expression was associated with grade $(p=0.046)$ and anatomical region of the examined malignancies (transglottic, $p=0.047$ ). Interestingly, high BCL2 expression levels were strongly associated with radiotherapy-based only regimens $(p=0.01)$ in corresponding patients. Conclusion: BCL2 overexpression was found to be correlated with an aggressive phenotype (advanced grade of differentiation) in LSCC, also demonstrating a potential selective anatomic localization (transglotic region). Additionally, BCL2 overexpression appears to be a negative regulator for successful radiotherapy implementation by reducing the apoptotic process in patients.
\end{abstract}

Apoptosis refers to genetically programmed cell death mediated by a complex of proteins which positively or negatively influence the apoptotic cascade (1). Two main

Correspondence to: Evangelos Tsiambas, Cytopathologist, MD, M.Sc., Ph.D., Lecturer Elect in Cytology, Medical School, University of Athens 17 Patriarchou Grigoriou E' Street, Ag. Paraskevi, 15341 Athens, Greece. Mobile: +30 6946939414, e-mail: tsiambasecyto@yahoo.gr

Key Words: Carcinoma, larynx, apoptosis, BCL2, DIA. pathways are involved in apoptosis: intrinsic and extrinsic. In both these, several proteins are characterized as inducers or inhibitors of apoptosis (2). Concerning the intrinsic pathway, mitochondrial proteins, prominently cytochrome $c$ from the inter-membrane space of the organelle, are responsible for regulating apoptotic signal transduction. Cytochrome $c$ in cytoplasm activates caspases (especially caspases 3, 8 and 9) under the control of p53 and B-cell lymphoma (BCL) proteins (3). BCL proteins were initially identified and cloned in studies of chromosomal translocations involving chromosomes 14 and 18 [t(14;18)] in follicular lymphomas, but its expression is not specific only to this genetic aberration $(4,5)$. BCL2 (gene locus:18q21.33, encoded protein product of $25 \mathrm{kDa}$ ) is the main member of the $\mathrm{BCL}$ protein family. It is located on the outer mitochondrial membrane acting mainly as an anti-apoptotic factor suppressing and blocking apoptotic signal transduction. It consists of two isoforms (1 and 2) which demonstrate specific anti-apoptotic functions. Concerning head and neck squamous cell carcinomas and especially laryngeal squamous cell carcinoma (LSCC), aberrant BCL2 expression seems to modify the chemoradiotherapy response rates in sub-groups of patients combined, or not, with the expression of other proteins, such as p27 and NOTCH2 $(6,7)$.

In the current study, we analyzed BCL2 protein expression levels in LSCC tissue sections, correlating their digitized staining intensity levels with the corresponding clinicopathological features.

\section{Materials and Methods}

Study group. For the purposes of our study, 50 archival, formalinfixed and paraffin-embedded tissue specimens of surgically resected, histologically confirmed primary LSCCs were used. The specimens were from 45 male and 5 female patients all smokers without a positive DNA test or a clear history of human papilloma virus infection. The hospital Ethics Committee consented to the use of these tissues at the Department of Pathology, Hippocration 
Hospital, University of Athens, Athens, Greece for research purposes, according to the World Medical Association Declaration of Helsinki guidelines (2008). According to their clinical status, patients were treated by chemotherapy (cisplatin-based), radiotherapy, or combined chemoradiotherapy regimens. The medial survival was 43 months.

The tissue samples were fixed in $10 \%$ neutral-buffered formalin. Hematoxylin and eosin-stained slides of the corresponding samples were reviewed for confirmation of histopathological diagnoses. All lesions were classified according to the histological typing criteria of World Health Organization (WHO) (8). Clinicopathological data of the examined cases are given in Table I.

Antibodies and immunohistochemistry (IHC). IHC for BCL2 marker expression was carried out on $4-\mu \mathrm{m}$ serial sections of the corresponding tissue blocks using an automated staining system (I 6000; Biogenex, San Ramon, CA, USA). The corresponding slides were deparaffinized and rehydrated. Sections were enzyme digested for $10 \mathrm{~min}$ at $37^{\circ} \mathrm{C}$. The EnVisionTM+ detection system (Dako, Glostrup, Denmark) was used for the detection steps. Blocking solution was applied to the slides for $10 \mathrm{~min}$, followed by incubation for $1 \mathrm{~h}$ using mouse monoclonal anti-BCL2 (clone NCLL-BCL-2, 1:100 dilution; Novocastra/Leica Biosystems Ltd, Newcastle, UK) was applied for $30 \mathrm{~min}$ at room temperature. Following incubation with the secondary antibody for $10 \mathrm{~min}$, diaminobenzidine-tetrahydrocloride $(0.03 \%)$ containing $0.1 \%$ hydrogen peroxide was applied as a chromogen and slides were incubated for $5 \mathrm{~min}$. Sections were counterstained, dehydrated and cover-slipped. For negative control slides, the primary antibody was omitted. Microscopically normal archival tonsil and normalappearing laryngeal epithelial tissue sections expressing BCL2 protein were considered positive controls. Membranous and diffuse cytoplasmic/peri-nuclear cellular staining patterns were considered acceptable for essential BCL2 expression (Figure 1A-C). Protein expression levels were evaluated quantitatively by implementing a digital image analysis (DIA) protocol.

DIA assay. BCL2 protein expression levels were evaluated quantitatively by calculating the corresponding staining intensity levels. DIA was performed using a semi-automated system (CX-31 Microscope - Olympus, Melville, NY, USA; Digital camera - Sony, Tokyo, Japan; Windows XP/NIS-Elements Software AR v3.0 Nikon Corp, Tokyo, Japan). Areas of interest per tissue section were identified (five optical fields at $\times 100$ magnification) and filed in a digital database as snapshots. Measurements were performed by implementing a specific macro assessing diffuse and focal membranous/cytoplasmic protein expression. Based on an algorithm, normal tissue sections (control) were measured independently and compared to the corresponding values in malignant tissue sections. A broad spectrum of continuous greyscale values (0-255) in red-green-blue (RGB) analysis was available for discriminating different protein expression levels (Figure 1D). Values decreasing to 0 represent progressive overexpression of the marker, whereas values increasing to 255 show a progressive loss of staining intensity. Results and DIA values are given in Table I.

Statistical analysis. Descriptive statistics were performed. Associations between variables including protein expression levels, and clinicopathological parameters such as gender, tumor grade and stage, anatomic location, and alcohol consumption were performed using Pearson chi-square test estimated along with its $99 \%$ confidence intervaI (CI) and Spearman correlation coefficient (SPSS v20; SPSS Inc, IBM, Armonk, NY, USA). Two-tailed values of $p \leq 0.05$ were considered statistically significant. Results and correlations ( $p$-values) are described in Table I.

\section{Results}

According to the protein DIA procedure, all the examined cases were evaluated properly demonstrating different BCL2 expression levels. High BCL2 protein expression levels (dark staining intensity) were observed in 21/50 (42\%) LSCC tissue sections, whereas the remaining cases $(n=29)$ demonstrated low expression (bright staining intensity). Overall BCL2 expression was associated with increased grade $(p=0.046)$ and transglottic anatomical location $(p=0.047)$. Interestingly, high BCL2 expression levels were strongly associated with regimens based on radiotherapy only $(p=0.01)$, whereas overall therapeutic strategies were not correlated with protein expression levels $(p=0.729)$. No other statistical correlations were identified concerning the other clinicopathological parameters (gender: $p=0.926$, stage: $p=0.252$, alcohol consumption status: $p=0.392$, overall survival in months: $p=0.08$ ).

\section{Discussion}

Deregulation of apoptotic pathways is a frequent event in head and neck squamous cell carcinoma, leading to increased resistance rates to specific radio-chemotherapeutic regimens in patients combined with advanced stage (lymph node metastases) disease (9). Especially focused on LSCCs, recently published data have shown that mitochondrial-based apoptotic proteins seem critically to affect prognosis in subgroups of patients with specific characteristics. A study group co-analyzed $B C L 2$ and BCL2-associated $\mathrm{X}(B A X)$ mRNA expression levels by implementing a real-time quantitative polymerase chain reaction protocol (10). They concluded that increased $B A X$ expression combined with decreased BCL2 (high $B A X / B C L 2$ mRNA expression ratio) was correlated with longer disease-free and overall survival of patients with a low tumor stage (negative lymph nodes at the time of diagnosis). For these reasons, they proposed the $B A X / B C L 2$ mRNA ratio as a potentially reliable biomarker in predicting biological behavior in patients with LSCC. Additionally, another experimental study analyzing the effect of combined acetazolamide and cisplatin chemotherapy application in Hep-2 laryngeal cell cultures showed increased levels of caspase-3 and BAX expression, whereas BCL2 protein levels were reduced (11).

In the current study, we analyzed BCL2 protein expression by IHC on tissue sections of LSCCs estimating the levels of its staining intensity by implementing a DIA protocol. High BCL2 protein expression levels were observed in a significant 

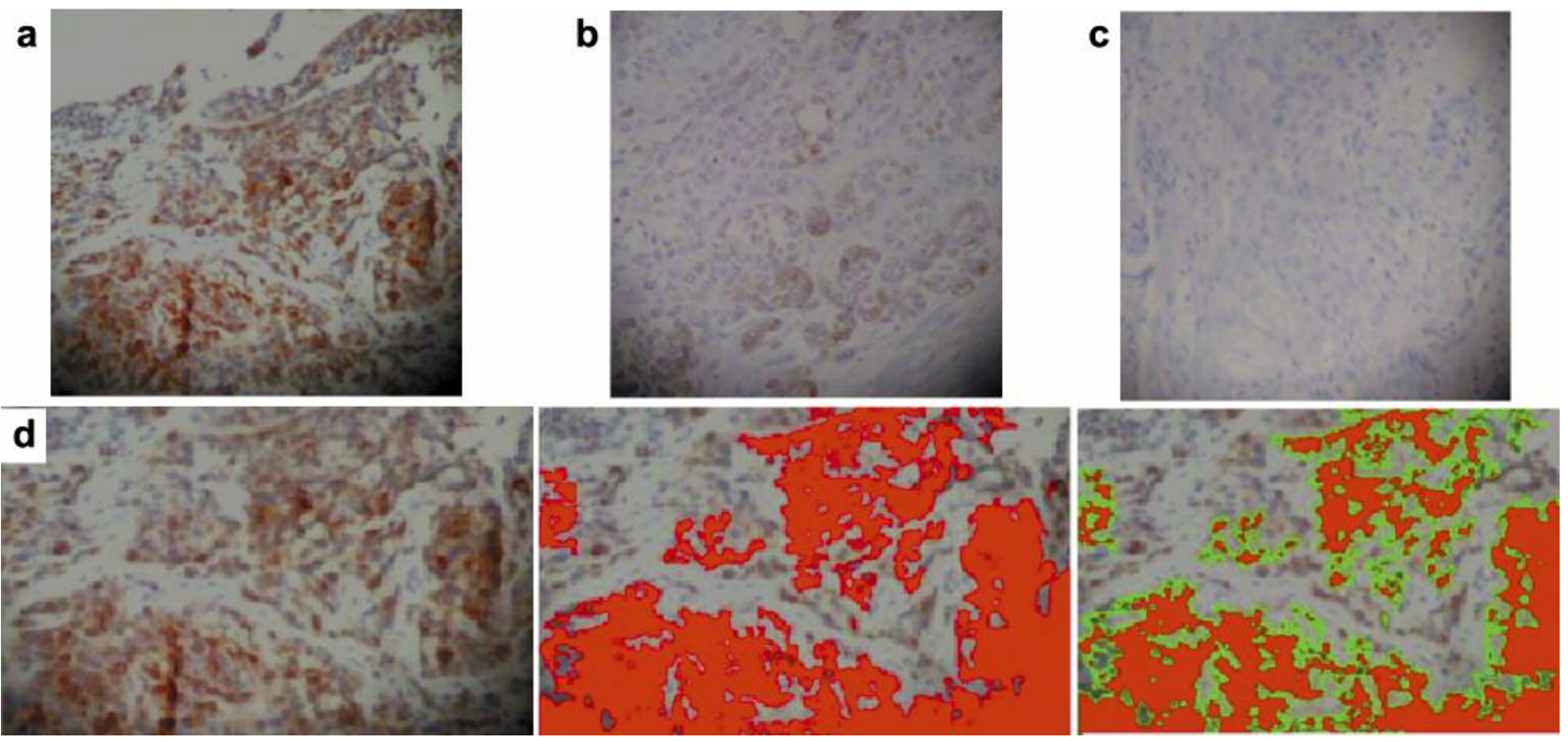

Figure 1. B-Cell lymphoma 2 (BCL2) protein expression patterns in laryngeal squamous cell carcinoma tissues. High (A), medium (B), and low (C) staining levels of the marker. Note membranous and diffuse cytoplasmic/peri-nuclear cellular staining (anti-BCL2, diaminobenzidinetetrahydrocloride stain; original magnification 100X). D: Implementation of digital image analysis protocol for estimating BCL2 protein expression levels. Progressive digitized snapshot of a microscopic field is analyzed by a macro focused on staining intensity values (0-255 RGB). Red areas show expression levels of the marker. The final value is the result of a synthetic approach of all stained pixels (original magnification 100x).

Table I. Clinicopathological parameters and total B-cell lymphoma 2 (BCL2) expression in patients with laryngeal squamous cell carcinoma ( $n=50)$.

\begin{tabular}{|c|c|c|c|c|}
\hline \multirow[b]{2}{*}{ Clinicopathological parameter } & \multirow[b]{2}{*}{ Frequency } & \multicolumn{2}{|c|}{ BCL2 expression, n (\%) } & \multirow[b]{2}{*}{$p$-Value } \\
\hline & & Overexpression $21 / 50(42 \%)$ & Low expression $29 / 50(58 \%)$ & \\
\hline \multicolumn{5}{|l|}{ Gender } \\
\hline Male & $45(90 \%)$ & $19 / 50(38 \%)$ & $26 / 50(52 \%)$ & 0.926 \\
\hline Female & $5(10 \%)$ & $2 / 50(4 \%)$ & $3 / 50(6 \%)$ & \\
\hline \multicolumn{5}{|l|}{ Anatomical region } \\
\hline Supraglottis & $5(10 \%)$ & $0 / 50$ & $5 / 50(10 \%)$ & 0.047 \\
\hline Transglottis & $45(90 \%)$ & $21 / 50(42 \%)$ & $24 / 50(48 \%)$ & \\
\hline \multicolumn{5}{|l|}{ Grade } \\
\hline 1 & $4(8 \%)$ & $1 / 50(2 \%)$ & $3 / 50(6 \%)$ & 0.046 \\
\hline 2 & $19(38 \%)$ & $3 / 50(6 \%)$ & $16 / 50(32 \%)$ & \\
\hline 3 & $27(54 \%)$ & $17 / 50(34 \%)$ & $10 / 50(20 \%)$ & \\
\hline \multicolumn{5}{|l|}{ Stage } \\
\hline II & $4(34 \%)$ & $1 / 50(2 \%)$ & $3 / 50(6 \%)$ & 0.252 \\
\hline III & $25(50 \%)$ & $8 / 50(16 \%)$ & $17 / 50(34 \%)$ & \\
\hline IV & $21(42 \%)$ & $12 / 50(24 \%)$ & $9 / 50(18 \%)$ & \\
\hline \multicolumn{5}{|l|}{ Alcohol consumption } \\
\hline Yes & $43(86 \%)$ & $17 / 50(34 \%)$ & $26 / 50(52 \%)$ & 0.392 \\
\hline No & $7(14 \%)$ & $4 / 50(8 \%)$ & $3 / 50(6 \%)$ & \\
\hline \multicolumn{5}{|l|}{ Treatment } \\
\hline Chemotherapy & $17(34 \%)$ & $6 / 50(12 \%)$ & $11 / 50(22 \%)$ & 0.729 \\
\hline Radiotherapy & $2(50 \%)$ & $2 / 50(4 \%)$ & $0 / 50$ & 0.01 \\
\hline Combined therapy & $31(42 \%)$ & $11 / 50(22 \%)$ & $20 / 50(40 \%)$ & \\
\hline \multicolumn{5}{|l|}{ Survival } \\
\hline 0-30 Months & $28(56 \%)$ & $14 / 50(28 \%)$ & $14 / 50(52 \%)$ & 0.08 \\
\hline$>30$ Months & $22(44 \%)$ & $7 / 50(8 \%)$ & $15 / 50(6 \%)$ & \\
\hline
\end{tabular}

Overexpression: Moderate to high expression, staining intensity values $\leq 137$ in $100 \%$ of the examined nuclei (spectrum between 76 and 137 ); Low expression: staining intensity values $>144$ in $100 \%$ of the examined nuclei (spectrum between 145 and 187). 
proportion of the examined tissues. Overall, BCL2 expression was associated with increasing grade and transglottic anatomical region of LSCC. Furthermore, high BCL2 expression levels were associated with use of radiotherapy alone. No other statistical correlations were identified concerning the other clinicopathological parameters. Referring to radiotherapy response rates, a recently published molecular study based on a polymerase chain reaction/restriction fragment length polymorphism analysis protocol identified a specific $B C L 2$ polymorphism (BCL2$938 \mathrm{C}>\mathrm{A}$ ) associated with worse disease-specific survival (12). They suggested that this polymorphism should be considered as a sensitive genetic biomarker for predicting the genetic profile of radiotherapy response in these patients. Similarly, epigenetic changes, including methylation modifications and micro-RNA (miR) deregulation, affect BCL2 protein expression. Focused on aberrant miR expression in LSCCs, a molecular study identified a specific $m i R-34 c$ interaction with BCL2 (13). Based on a complex molecular analysis including reverse transcription-quantitative polymerase chain reaction and western blot analysis, they measured $B C L 2$ mRNA and protein expression levels in transfected M4e cells. They concluded that $m i R$ - $34 c$ induces apoptosis and inhibits the viability of M4e cells by targeting BCL2. Concerning novel genetic factors that modify BCL2 expression by down-regulating its activity and enhancing the apoptotic process in LSCCs, a study group analyzed the human esophageal cancer related gene 4 (ECRG4) and considered it a potential tumor suppressor and inhibitor of BCL2 (14). Similarly, another study group investigated the role of Beclin1 protein expression in LSCC cell cultures. They concluded that the molecule's activity is modified by in vitro application of cisplatin. In fact, BECLIN1 was found to promote cisplatin-induced apoptosis in Hep-2 laryngeal carcinoma cells by BCL2-modulated autophagy (15). These previous studies underline the significance of BCL2 deregulation at the DNA and mRNA levels, explaining its aberrant protein expression in LSSC tissue sections shown by IHC implementation. Moreover, in this study we performed a DIA protocol, as an innovative, accurate and fast technique for objectively estimating the corresponding levels of BCL2 protein expression, as we have already implemented in evaluating expression of other molecules (16).

\section{Conclusion}

BCL2 overexpression is correlated with an aggressive phenotype (advanced grade of differentiation) in LSCC, also demonstrating a potential selective anatomic localization, namely the transglottic region. BCL2 overexpression is a negative regulator for successful radiotherapy implementation by reducing the apoptotic process. For this reason, development of synthetic agents or application of natural anti-
BCL2 agents, such as propolis, which demonstrated strong cytotoxic anticancer activity (17), is a very promising chemotherapeutic strategy for stabilizing and enhancing apoptosis in patients with LSCC.

\section{Conflicts of Interest}

The Authors declare no conflicts of interest in regard to this study. No financial support was granted.

\section{Authors' Contributions}

Aristeidis Chrysovergis: clinical advisor (case selection); Vasileios S. Papanikolaou: clinical advisor (case selection); Evangelos Tsiambas: researcher, article writing; Vasileios Ragos: academic advisor; Dimitrios Peschos: academic advisor; Efthymios Kyrodimos: academic advisor advisor, article writing.

\section{Acknowledgements}

The Authors acknowledge the significant scientific contribution of Eleftherios Stavrakas, MEng, MSc, PMI-RMP, CEng, PRINCE2 Consultant, Germany, for his extensive statistical analysis.

\section{References}

1 Arumugam A and Razis AF: Apoptosis as a mechanism of the cancer chemopreventive activity of glucosinolates: A review. Asian Pac J Cancer Prev 19(6): 1439-1448, 2018. PMID: 29936713. DOI: 10.22034/APJCP 2018.19.6.1439

2 Chung C: Restoring the switch for cancer cell death: Targeting the apoptosis signaling pathway. Am J Health Syst Pharm 75(13): 945-952, 2018. PMID: 29759975. DOI: 10.2146/ ajhp170607

3 Dorstyn L, Akey CW and Kumar: New insights into apoptosome structure and function. Cell Death Differ 25(7): 1194-1208, 2018. PMID: 29765111. DOI: 10.1038/s41418-017-0025-Z

4 Von Haefen $\mathrm{C}$, Wieder $\mathrm{T}$ and Gillissen: Ceramide induces mitochondrial activation and apoptosis via a BAX-dependent pathway in human carcinoma cells. Oncogene 21(25): 40094019, 2002. PMID: 12037683. DOI: $10.1038 / \mathrm{sj}$. onc1205497

5 Pezzella F, Tse AGD and Cordell JL: Expression of the BCL-2 oncogene protein is not specific for the 14;18 chromosomal translocation. Am J Pathol 137(2): 225-232, 1990. PMID: 2201196.

6 Moreno-Galindo C, Hermsen M, García-Pedrero JM, Fresno MF, Suárez C, and Rodrigo JP: p27 and BCL2 expression predicts response to chemotherapy in head and neck squamous cell carcinomas. Oral Oncol 50(2): 128-134, 2014. PMID: 24239278. DOI: 10.1016/j.oraloncology 2013.10.018

7 Zou Y, Fang F, Ding YJ, Dai MY, Yi X, Chen C, Tao ZZ, and Chen SM: Notch 2 signaling contributes to cell growth, antiapoptosis and metastasis in laryngeal squamous cell carcinoma. Mol Med Rep 14(4): 3517-3524, 2016. PMID: 27572051. DOI: $10.3892 / \mathrm{mmr} 2016.5688$

8 Barnes L, Eveson JW, Reichart P, and Sidransky D: Pathology and Genetics: Head and Neck Tumours. WHO IARC Press, Lyon, France, pp. 118-130, 2005. 
9 Takes RP, Baatenburg de Jong RJ and Wijffels K: Expression of genetic markers in lymph node metastases compared with their primary tumours in head and neck cancer. J Pathol 194: 298-302, 2001. PMID: 11439361. DOI: 10.1002/1096-9896(200107) 194:3<298::AID-PATH900>3.0.CO;2-Q

10 Giotakis AI, Kontos CK, Manolopoulos LD, Sismanis A, Konstadoulakis MM and Scorilas A: High $B A X / B C L 2$ mRNA ratio predicts favorable prognosis in laryngeal squamous cellcarcinoma, particularly in patients with negative lymph nodes at the time of diagnosis: Clin Biochem 49(12): 890-896, 2016. PMID: 27129795. DOI: 10.1016/j.clinbiochem 2016.04.010

11 Gao H, Dong H, Li G and Jin H: Combined treatment with acetazolamide and cisplatin enhances chemosensitivity in laryngeal carcinoma Hep-2 cells: Oncol Lett 15(6): 9299-9306, 2018. PMID: 29928333. DOI: 10.3892/ol2018.8529

12 Agostini LP, Stur E, Garcia FM, Ventorim DP, Dos Reis RS, Dettogni RS, Dos Santos EVW, Peterle GT, Maia LL, Mendes SO, de Carvalho MB, Tajara EH, de Paula F, Dos Santos M, da Silva AMA, and Louro ID: $A T M, B C L 2$, and $T G F \beta$ gene polymorphisms as radiotherapy outcome biomarkers in head and neck squamous cell carcinoma patients. Genet Test Mol Biomarkers 21(12): 727-735, 2017. PMID: 29135311. DOI: $10.1089 / \mathrm{gtmb} 2017.0180$

$13 \mathrm{Li} \mathrm{R}$, Zhang $\mathrm{H}$ and Zheng $\mathrm{X}$ : $m i R-34 c$ induces apoptosis and inhibits the viability of M4e cells by targetingBCL2. Oncol Lett 15(3): 3357-3361, 2018. PMID: 29435079. DOI: 10.3892/ ol2017.7640
14 Jia J, Dai S, Sun X, Sang Y, Xu Z, Zhang J, Cui X, Song J and Guo X: A preliminary study of the effect of ECRG4 overexpression on the proliferation and apoptosis of human laryngeal cancer cells and the underlying mechanisms. Mol Med Rep 12(4): 5058-5064, 2015. PMID: 26165988. DOI: 10.3892/ mmr2015.4059

15 Yang M, Yang XM and Yin DH: BECLIN1enhances cisplatininduced apoptosis via BCL2-modulated autophagy in laryngeal carcinoma cells Hep-2. Neoplasma 65(1): 42-48, 2018. PMID: 29322787. DOI: 10.4149/neo_2018_161102N528

16 Mastronikolis NS, Tsiambas E, Papadas TA, Karameris A, Ragos V, Peschos D, Mastronikolis SN, Liatsos C, Armata IE, and Fotiades PP: Deregulation of PTEN expression in laryngeal squamous cell carcinoma based on tissue microarray digital analysis. Anticancer Res 37(10): 5521-5524, 2017 PMID: 28982865. DOI: 10.21873/anticanres11983

17 Frión-Herrera Y, Díaz-García A, Ruiz-Fuentes J, RodríguezSánchez H and Maurício Sforcin J: Mechanisms underlying the cytotoxic effect of propolis on human laryngeal epidermoid carcinoma cells. Nat Prod Res 32(17): 2085-2091, 2018. PMID: 28783983. DOI: $10.1080 / 14786419.2017 .1363749$

Received January 9, 2019

Revised February 15, 2019 Accepted February 19, 2019 